Journal of Case Reports 2018;8(4):232-235

\title{
Therapeutic Heart Catheter through an ECMOArterial Line: Non-conventional way Made Possible
}

\author{
Karam Issa, Bernward Lauer, Moritz Laubscher, Marc Alexander Ohlow \\ Department of Cardiology, Zentralklinik Bad Berka, Bad Berka, Germany.
}

\author{
Corresponding Author: \\ Dr. Karam Issa \\ Email: karam_issa@hotmail.com
}

This is an Open Access article distributed under the terms of the Creative Commons Attribution License (creativecommons.org/ licenses/by/3.0).

Received Accepted

Published

January 29, 2018

September 10, 2018

October 10,2018

\begin{abstract}
Background: Coronary angiography is an important diagnostic tool in patients with acute coronary syndrome. Case Report: We report a case of a 39-years-old male patient with severe peripheral arterial disease, who presented to us in cardiogenic shock. To establish an arterial line was the challenging corner stone for both extracorporeal membrane oxygenation and for heart catheter. Conclusion: We succeeded to ensure a safe and effective arterial way to catheterize the patient diagnostically and therapeutically through direct puncture of the ECMO arterial cannula.
\end{abstract}

Keywords: Cardiogenic Shock, Coronary Angiography, Coronary Vessels, Extracorporeal Membrane Oxygenation, Peripheral Arterial Disease, Percutaneous Coronary Intervention.

\section{Introduction}

Extracorporeal membrane oxygenation (ECMO) is increasingly used over the recent years as a method of cardiovascular resuscitation; it became invaluable tool in the care of patients with severe cardiac dysfunction refractory to conventional management [1]. Patients on ECMO can present a real challenge for vascular access depending upon cannulation and invasive monitoring sites. The non-pulsatility of ECMO flow and relatively low pressure can make it difficult to puncture an artery using a percutaneous approach. Multiple percutaneous cannulation attempts can be a precursor to bleeding complications as these patients are fully heparinized to achieve an activated clotting time of 150 to 250 seconds. To avoid this scenario, we followed a new technique to gain arterial access for coronary angiography.

\section{Case Report}

A 39-years-old male patient was transferred to our emergency department in a picture of acute coronary syndrome with typical anginal chest pain radiating to the left shoulder and ischemic ECG changes (ST-segment elevation in V1-V4). The patient was known to be a heavy smoker with positive family history (mother with myocardial infarction). Because of respiratory failure with persistently acidotic blood gases, all corrective pharmacological maneuvers did not withstand his status. Consequently, the patient was electively intubated for better respiratory drive. ChestX-ray showed normal cardiac configuration with mildly obliterated left cardiophrenic angle and hence mildly congested lunge fields. The echocardiography showed a massively impeded left ventricular ejection fraction (EF 10\%), but normal right ventricular function with no concomitant valve pathologies or evidence of aortic dissection.

Because of his hemodynamically unstable condition and to re-establish sufficient cardiac output we decided to connect him on a venoarterial ECMO. In the cardiac catheterization laboratory, multiple unsuccessful attempts to introduce an arterial access through the right and left femoral arteries obliged us to utilize the right 
axially artery as an arterial ECMO cannula input way. A 17 F (French) ECMO arterial cannula (Co. MAQUET GETINGE GROUP, Rastatt Germany) was successfully instituted via a right axially artery cut down in the cardiac catheterization laboratory. The right femoral vein was successfully cannulated through a $25 \mathrm{~F}$ ECMO venous cannula (Co. MAQUET GETINGE GROUP, Rastatt Germany). After establishment of ECMO support and achieving an adequate circulatory volume flow (4.2 liters/min) and hemodynamics [mean arterial pressure (MAP) $75 \mathrm{mmHg}$, venous saturation above $68 \%$ ], the patient was prepared for coronary angiography. Inspite of the previous unsuccessful trials to obtain an arterial access, we insisted on diagnosing this failed trials through respective angiography in the right femoral artery which showed atherosclerotic stenotic and obstructed axis post the aortic bifurcation denoting Leriche syndrome [Fig.1].

To perform the coronary angiography, we punctured the left radial artery. However, it was not possible to reach the heart due to occlusion of the left subclavian artery [Fig.2]. Before hope fades away, aroused the idea of using the ECMO arterial cannula to catheterize the patient. For safety measures, we decided to test this approach on a separate non utilized part of the ECMO line. We punctured the arterial line with a conventional puncture needle. Through this needle we introduced a Teflon wire then replaced the needle with a 5 F-sheath (AVANTI+ Cordis) which was properly implanted [Fig.3]. Consequently, the same maneuver was applied safely and successfully at the arterial line of the ECMO [Fig.4,5].

Diagnostic coronary angiography was performed through the ECMO arterial line showing $65 \%$ stenosis in the proximal left main and $80 \%$ stenosis in the proximal LAD [Fig.6], which were successfully stented with two drug eluting stents [Fig.7]. Moreover, we were able to take multiple left ventricular myocardial biopsies for myocarditis workup, which was lately diagnosed to be negative.

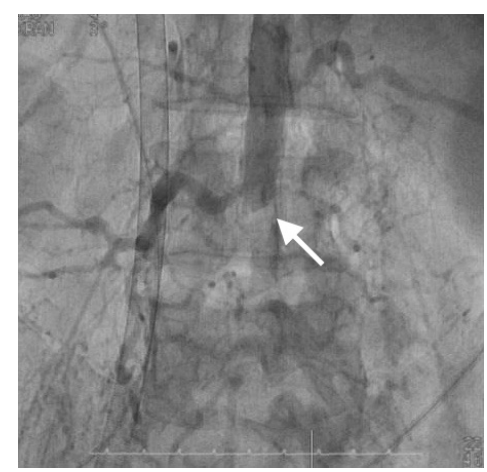

Fig.1: Angiography of descending aorta showing Leriche syndrome.

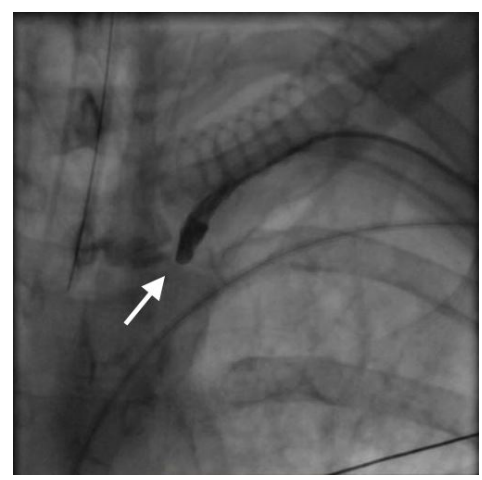

Fig.2: Angiography of the left subclavian artery showing total occlusion.

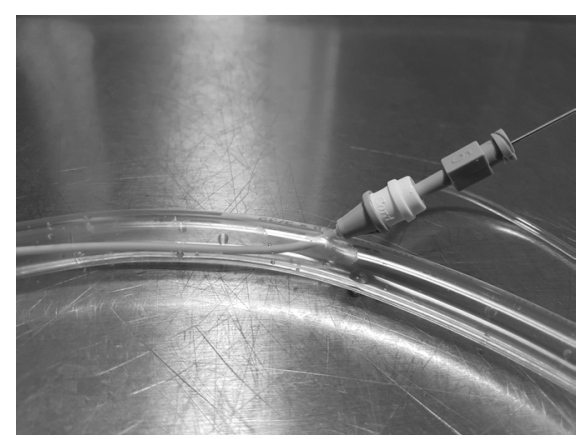

Fig.3: Trial of the 5 F-sheath insertion to the ECMO line.

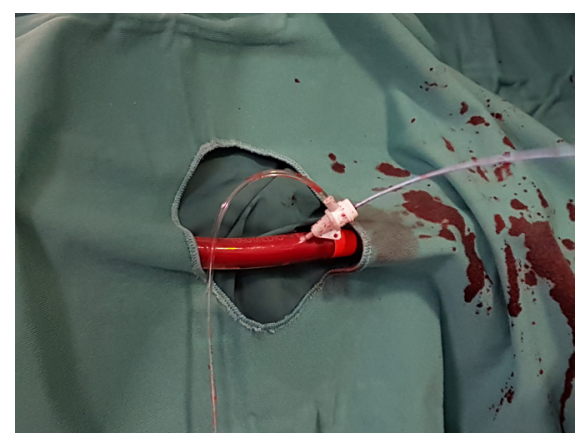

Fig.4: The 5 F-sheath inserted in the ECMO arterial line. 
Due to the lack of an adequate arterial line for continuous invasive hemodynamic monitoring we decided to leave our $5 \mathrm{~F}$-sheath in place for further aid in the ICU. The patient was safely transferred to the ICU in a hemodynamic stable state. The gradual patient's clinical and echocardiographical (EF 35\% after 72 hours) stabilization enabled us to reduce the inotropic support and wean the ECMO. The patient was on the fifth day ECMO and inotropes free in the ICU.

\section{Discussion}

Patients presenting with acute coronary syndrome are associated with higher risk of ischemic complications and death [2]. An invasive strategy can improve the prognosis. Since a long time coronary angiography, through radial, brachial or femoral arterial access has been the standard procedure for both diagnosis and therapy [2]. With the lake of these standard accesses it would more difficult and challenging to perform. Our case illustrates that coronary angiography can be successfully performed via the extracorporeal circuit on ECMO, as only intra-arterial access, obviating the need for additional vascular access. The idea was previously tested on non-functional part of the ECMO line to ensure its feasibility. Live application of this manoeuvre was safe and easy, the passage of the guide through the ECMO cannula occurred with no major difficulties and the stent navigability was not harmed. Practically, this technique could be applied in ECMO setup with no need to further or specific cannulation system. This technique does not need any anatomic specific considerations as for example; a straight way to the aortic root. It will be may be less suitable for small children where the French scale size of the angiography catheter is around half of the French scale size of the ECMO cannula, and the flow may be severely restricted [3]. For the group of patients like our case with peripheral vascular insufficiency, the access alternatives are a direct arterial access via ECMO by insertion of a hemostatically valved

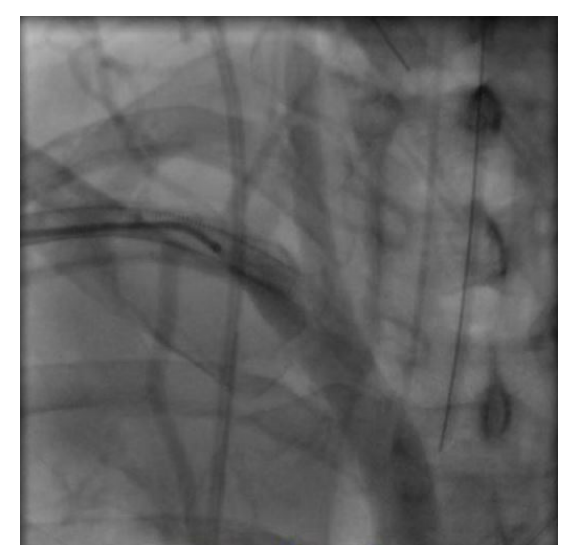

Fig.5: Successful angiography of the brachio-cephalic-trunk through the ECMO arterial line.

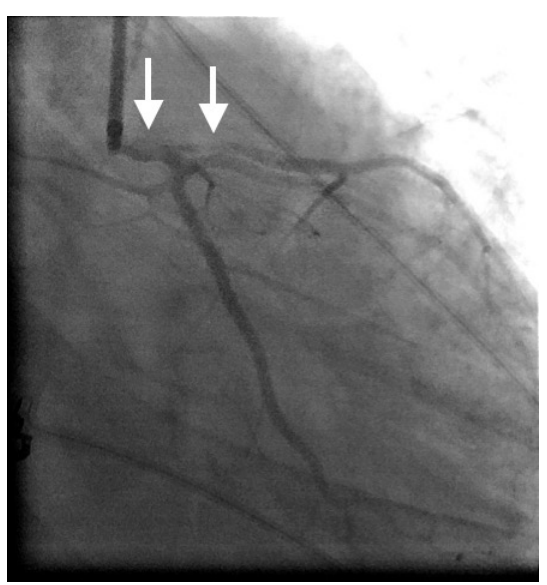

Fig.6: Coronary angiography showing significant stenosis in the proximal LAD and proximal LM.

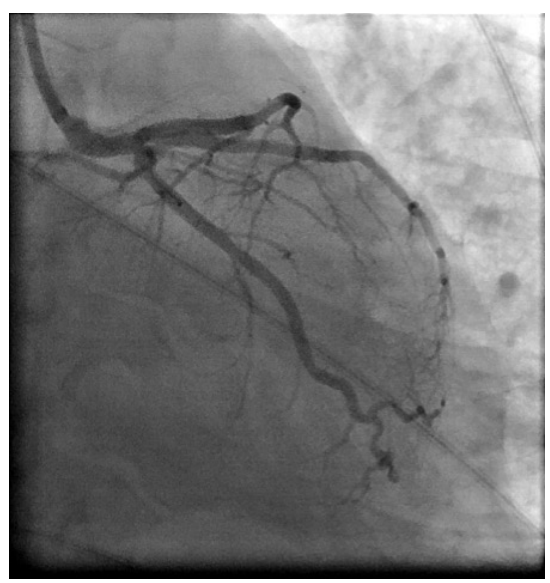

Fig.7: Coronary angiography of the LAD and LM after percutaneous coronary intervention. 
Y connector near to the femoral arterial cannula [3], which was not available in our institution, an anterograde access through transseptal route [4] and a transcaval access through femoral vein via a crossing system [5], which was performed on over 200 patients in the USA. These alternatives are more useful in elective patients with enough time to plan an angiography, furthermore this was not possible in an emergent case because the lake of necessary equipment and time.

\section{Conclusion}

Performing coronary angiography through the ECMO by direct puncture of the arterial cannula to allow access for catheterization with failed percutaneous access site because of peripheral vascular insufficiency is a challenge, but it's possible as a last resort. It was safe, effective and performed with no complications. We were able to gain the benefits not only of catheterizing the patient but also of obtaining biopsies and lying an invasive pressure monitoring line.

Contributors: KI: manuscript writing, patient management; BL, ML, MAO: manuscript editing, patient management. KI will act as guarantor. All authors approved the final version of this manuscript.

Funding: None; Competing interests: None stated.

\section{References}

1. Shekar K, Mullany DV, Thomson B, Ziegenfuss M, Platts DG, Fraser JF. Extracorporeal life support devices and strategies for management of acute cardiorespiratory failure in adult patients: a comprehensive review. Crit Care. 2014;18:219.

2. Ohlow MA, Secknus MA, von Korn H, Neumeister A, Wagner A, Yu J, et al. Incidence and outcome of femoral vascular complications among 18,165 patients undergoing cardiac catheterisation. Int $\mathrm{J}$ Cardiol. 2009; 135:66-71.

3. Thuys C, MacLaren G, d'Udekem Y, MD, Eastaugh L. Vascular access for pediatric coronary angiography on extracorporeal membrane oxygenation. World J Pediatr Congenit Heart Surg. 2015;6:126-129.

4. Wang R, Stadler Souza N, Lima A, Vinícius de Freitas Moreira M. Transseptal coronary angioplasty in patients with severe peripheral vascular disease. Arq Bras Cardiol. 2013;101:11-14.

5. Lederman RJ, Greenbaum AB, Rogers $\mathrm{T}$ Khan JM, Fusari M, Chen MY. Anatomic Suitability for Transcaval Access Based on Computed Tomography. JACC Cardiovasc Interv. 2017;10:1-10. 